Matsuo M., Yokoyama M., Umemura K., Gril J., Yano H., Kawai S. (2010) Color changes in wood during heating: kinetic analysis by applying time-temperature superposition method, Applied Physics A, Materials Science \& Processing, vol. 99, 1, p. 47-52. DOI 10.1007/s00339-010-5542-2 (Author's copy)

\title{
Color changes in wood during heating: kinetic analysis by applying a time-temperature superposition method
}

\author{
Miyuki Matsuo**1, Misao Yokoyama ${ }^{1}$, Kenji Umemura ${ }^{1}, J_{0 s e p h}$ Gril $^{1,3}$, \\ Ken'ichiro Yano², Shuichi Kawai ${ }^{1}$
}

\footnotetext{
${ }^{1}$ Research Institute for Sustainable Humanosphere, Kyoto University, Gokasho, Uji, Kyoto 611-0011, Japan

${ }^{2}$ Tokyo University of the Arts, 12-8 Ueno Kouen, Taito-ku, Tokyo 110-8714, Japan

${ }^{3}$ Laboratoire de Mécanique et Génie Civil, Université Montpellier 2, CNRS, CC 048 Place Eugène Bataillon, 34095 Montpellier Cedex 5, France

*E-mail: matsuomiyuki@rish.kyoto-u.ac.jp
}

\begin{abstract}
This paper deals with the kinetics of the color properties of hinoki (Chamaecyparis obtusa Endl.) wood. Specimens cut from the wood were heated at $90-180^{\circ} \mathrm{C}$ as accelerated aging treatment. The specimens completely dried and heated in the presence of oxygen allowed us to evaluate the effects of thermal oxidation on wood color change. Color properties measured by a spectrophotometer showed similar behavior irrespective of the treatment temperature with each time scale. Kinetic analysis using the time-temperature superposition principle, which uses the whole data set, was successfully applied to the color changes. The calculated values of the apparent activation energy in terms of $L^{*}, a^{*}, b^{*}$, and $\Delta E^{*}{ }_{a b}$ were $117,95,114$, and $113 \mathrm{~kJ} / \mathrm{mol}$, respectively, which are similar to the values of the literature obtained for other properties such as the physical and mechanical properties of wood.
\end{abstract}

\section{Introduction}

Kinetic analysis has been successfully used to describe the temperature dependence of the reaction rate in chemical reactions. Applying kinetic analysis to wood and wood compounds allows one to predict the pyrolysis or deterioration of lignocellulosic materials. The kinetics of pyrolysis has been widely studied in the high temperature zone due to growing demand for lignocellulosic biomass as an alternative energy resource [1-5], while at the lower temperature zone they have been applied to the accelerated aging phenomena of materials to understand their natural aging [6-10]. Among the wood properties that change during low temperature heating, as reviewed by Esteves et al. [11], wood aging kinetics generally applies to properties such as weight loss, modulus of elasticity (MOE), modulus of rupture (MOR), and thermogravimetric data. However, these properties may require a destructive test on defect-free specimens, and the initial values that are needed, especially for 
comparison with naturally aging wood, are sometimes difficult to evaluate.

By contrast, color measurement is non-destructive and only requires small areas. Color properties are often used for kinetic analysis in the food industry not only for appearance but also for safety assessment, since they sometimes relate to deterioration [12-15]. Kinetic analysis applied to food color properties has successfully predicted food degradation. The color changes of wood during heat treatment have also been studied so as to obtain a good color appearance, to avoid undesirable color change during drying or heat treatment, and as a criterion of wood degradation during heating [16-20]. Heat treatment under both dry and wet conditions induces color changes in wood, especially darkening. However, few papers have reported on the kinetic analysis of color changes.

Heat treatment under dry conditions accompanies thermal oxidation of wood components, while heat treatment under wet conditions accompanies hydrolysis. Some researchers suggested that wood aging is a mild thermal oxidation at room temperature, based on their empirical data [6, 7, 21, 22]. Namely, wood aging might be explained as the same reaction as heat treatment under dry conditions. We have undertaken a preliminary study of the effects of temperature on wood color in order to improve our understanding of the process of natural wood aging, which bears many features in common with heat treatment under dry conditions. This study analyzes the color changes in wood by a kinetic approach based on the time-temperature superposition principle (TTSP) and the Arrhenius equation for accurate analysis. The specimens were completely dried to avoid the effects of hydrolysis and then treated at temperature levels well below the thermal decomposition point of wood. Our results indicate that the effects of thermal oxidation on color change can be estimated and that color change can therefore be predicted during natural aging, where almost only thermal oxidation occurs.

\section{Materials and methods}

\section{Specimens}

A 360-year-old hinoki (Chamaecyparis obtusa Endl.) from Kiso, Japan was used. The tree was harvested in 1988 and found to be corresponding to annual rings from A.D. 1629 to 1988, and the wood had been dried for 18 years under ambient conditions in a shed. A sample with homogeneous grain was carefully selected. Specimens were cut out with the dimensions of $120 \mathrm{~mm}$ (longitudinal direction $) \times 20 \mathrm{~mm}$ (radial direction) $\times 4 \mathrm{~mm}$ (tangential direction) from near the outermost part of the heartwood. The specimens were dried at $60^{\circ} \mathrm{C}$ in an air-circulating oven for 12 hours and then at room temperature in a desiccator with silica-gel and diphosphorus pentaoxide until a constant weight was obtained. Dried specimens were heated in an air-circulating oven at 4 temperature levels from 
90 to $180^{\circ} \mathrm{C}$ for a duration ranging from 0.5 hours to approximately 2 years. Table 1 shows the treatment times of the specimens at each treatment temperature. They were planned on assuming that a $10^{\circ} \mathrm{C}$ increase is equivalent to dividing the time by 2 [6].

TABLE 1 Treatment temperatures and time of accelerated aged wood samples. The treatment at $90^{\circ} \mathrm{C}$ is now in process, with the planned treatment time in parentheses.

\begin{tabular}{c|rrrr}
\hline & $90^{\circ} \mathrm{C}$ & $120^{\circ} \mathrm{C}$ & $150^{\circ} \mathrm{C}$ & $180^{\circ} \mathrm{C}$ \\
\hline & 256 & 32 & 4 & 0.5 \\
& 512 & 96 & 8 & 1 \\
& 1024 & & 16 & 2 \\
& 1536 & 192 & 24 & 3 \\
& 2560 & 320 & 40 & 5 \\
& & & 56 & 7 \\
Treatment & 5000 & & 80 & 10 \\
& 7296 & 768 & 96 & 12 \\
& 9216 & & 144 & 18 \\
& 12288 & & 192 & 24 \\
& 18432 & 2304 & 288 & 36 \\
& $(24576)$ & 3072 & 384 & 48 \\
& $(30720)$ & 3840 & 480 & 60 \\
& $(36864)$ & 4608 & & 72 \\
& $(43008)$ & & 672 & 84 \\
& $(49152)$ & 6144 & 768 & 96 \\
& $(61440)$ & 7680 & 960 & 120 \\
\hline
\end{tabular}

\section{Color measurement}

The color of the specimens was measured with a spectrophotometer (KONICA MINOLTA CM-2600d) using a D65 light source and an observed angle of 10 degrees. The sensor head of the spectrophotometer was $8 \mathrm{~mm}$ in diameter, which was sufficient to eliminate the influence on the color values of early and late wood variation, because the average annual ring width was $1.0 \mathrm{~mm}$ and the specimens had a homogeneous grain. The CIELAB color parameters $\left(L^{*}, a^{*}, b^{*}\right)$ were used to express the color change. The differences in parameters $\Delta L^{*}, \Delta a^{*}$, and $\Delta b^{*}$ and the total color differences $\Delta E^{*}{ }_{a b}$ were calculated using the following formulas [23]:

$$
\begin{aligned}
\Delta L^{*} & =L^{*}-L_{0}{ }^{*} \\
\Delta a^{*} & =a^{*}-a_{0}{ }^{*} \\
\Delta b^{*} & =b^{*}-b_{0}{ }^{*}
\end{aligned}
$$


$\Delta E_{a b}^{*}=\left[\Delta L^{* 2}+\Delta a^{* 2}+\Delta b^{* 2}\right]^{1 / 2}$

where $L^{*}$ is the lightness and $a^{*}$ and $b^{*}$ the color coordinates under any testing condition, and $L_{0} *$, $a_{0} *$, and $b_{0} *$ the corresponding reference values obtained as the average of 83 untreated specimens. $L_{0} *, a_{0} *$, and $b_{0} *$ were $82.0,7.20$, and 22.2 , respectively. Three to five specimens were tested under each condition. Three locations in each specimen were measured, and the average 9 to 15 values with standard deviations were calculated.

In addition, the color on the radial section of a matched wood block was measured along the radial direction to estimate the variations with time of wood formation. The color was measured at $10 \mathrm{~mm}$ intervals from the sapwood/heartwood boundary toward the pith for the range of $370 \mathrm{~mm}$, so that a sequence of color data along the time of wood formation was obtained.

\section{Results and discussion}

\section{Color changes during heat treatment}

Figure 1 shows the changes of $L^{*}, a^{*}, b^{*}$, and $\Delta E^{*}{ }_{a b}$ according to the duration and temperature of treatment. The decrease in $L^{*}$ at all temperatures indicates that the specimens became darker with the treatment time. This result corresponds to the increase in $\Delta E^{*}{ }_{a b}$, which is dominated by $L^{*}$ variations. The initial increase and following decrease of $a^{*}$ and $b^{*}$ indicate that the specimens initially became more red and yellow and then lost these colors. A similar behavior irrespective of treatment temperatures suggested that the same processes might cause the color changes. 

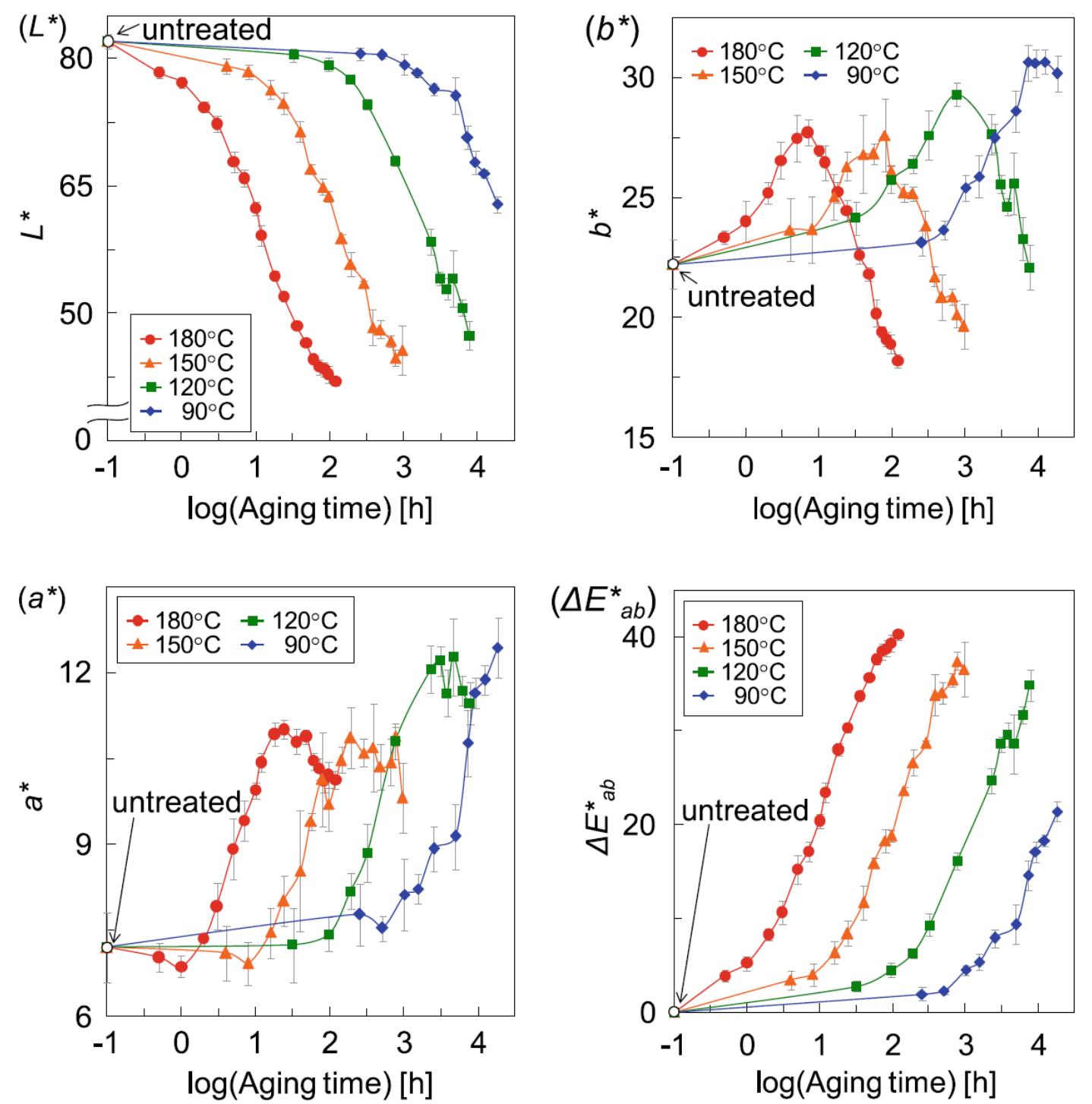

FIGURE 1 Changes in $L^{*}, a^{*}, b^{*}$, and $\Delta E$ during heat treatment as a function of time and temperature. $\left(\diamond: 90^{\circ} \mathrm{C}, \varpi: 120^{\circ} \mathrm{C}, \triangle: 150^{\circ} \mathrm{C}, \bullet: 180^{\circ} \mathrm{C}\right.$, error bar: standard deviation)

\section{Color changes with time of wood formation}

Although the color parameters changed slightly with the time of wood formation, there was little change in the color parameters, as indicated by the coefficient of variation being $<10 \%$ for color parameters $\left(L^{*}, a^{*}, b^{*}\right)$, within the range of $200 \mathrm{~mm}$, from which almost all specimens were cut out. These results indicate that little change responsible for color variations had occurred within the tree, and that the initial color could be considered as fairly homogeneous among the specimens.

\section{Kinetic analysis}


To predict the color changes that occur during natural aging, kinetic analysis was adopted. It is empirically known that a chemical reaction can be described by the Arrhenius equation:

$k=A \exp \left(-\frac{E_{a}}{R T}\right)$

where $k$ is the rate constant of the chemical reaction, $A$ the frequency factor, $E_{a}$ the apparent activation energy, $R$ the gas constant, and $T$ the absolute temperature of the reaction. The apparent activation energy is then obtained from the slope of the Arrhenius plot, which is the logarithm of the determined time versus the reciprocal of treatment temperature [8]. The regression line of the Arrhenius plot allows us to determine the reaction rate at any temperature.

When creating the Arrhenius plot, as mentioned above, only one processed data point is generally used from each accelerated aging temperature curve, eliminating most of the experimental points from the analysis. This elimination sometimes makes it difficult to accurately predict the changes in properties during natural aging. To determine the activation energy using all of the data, we assumed the applicability of the time-temperature superposition principle (TTSP) [24-27]. TTSP is a well-known concept that is frequently used to describe the mechanical and electrical relaxation behavior of polymers. The Arrhenius approach of TTSP has been successfully used for years in polymers to make predictions of aging under ambient conditions. According to the TTSP, both time and temperature are equivalent, i.e. material parameter values obtained for short times at a given temperature are identical to those measured for longer times at a lower temperature when the curves are shifted on a logarithmic time axis. The curves of the measured material parameter vs. logarithmic treatment time at different temperatures can be superimposed by proper scale changes on the log time axis. The shift distance along the logarithmic time axis is called the time-temperature shift factor $a_{T}$, given by

$a_{T}=t_{T} / t_{\text {ref }}$

where $t_{\text {ref }}$ is the test time at a reference temperature $T_{\text {ref }}$, and $t_{T}$ is the time required to give the same response at the test temperature $T$. Combining (3) and (4) gives

$a_{T}=\exp \left[\frac{E_{a}}{R}\left(\frac{1}{T}-\frac{1}{T_{r e f}}\right)\right]$

where both $T$ and $T_{\text {ref }}$ are absolute temperatures. Plotting $\ln \left(a_{T}\right)$ vs. $1 / T$ is another way to calculate $E_{a}$ and to predict changes in properties under ambient conditions.

A temperature of $180^{\circ} \mathrm{C}$ was chosen as $T_{\text {ref }}$ because it was convenient to express $a_{T}$ by extrapolating toward the ambient temperature for predicting natural aging. Some regressions were performed for $\Delta L^{*}, \Delta a^{*}, \Delta b^{*}$, and $\Delta E^{*}{ }_{a b}$ at $180^{\circ} \mathrm{C}$ expressed as a function of $t$. In other words, polynomial 
expressions, exponential functions, some sigmoidal functions, and some probability density functions were applied to the regression, and the functions giving the best fit were chosen judging from the coefficients of determination. The measured color properties at treatment temperatures lower than $180^{\circ} \mathrm{C}$ were superposed by $a_{T}$ on the time axis, and the constants of the regression curves were recalculated for the best fit. All parameters were estimated using a non-linear iterative curve-fitting method. A logistic function was used for $\Delta L^{*}$ and $\Delta E^{*}{ }_{a b}$ :

$$
f(x)=\frac{\alpha}{1+\beta \exp (-\gamma x)}
$$

where $f(x)$ is the color parameter, $x$ is $\log \left(t_{T} / a_{T} t_{\mathrm{ref}}\right)$, and $\alpha, \beta$, and $\gamma$ are constants.

A seventh-order polynomial expression was selected for $\Delta a^{*}$ and $\Delta b^{*}$ :

$$
g(x)=\sum_{k=3}^{7} p_{k} x^{k}
$$

where $g(x)$ is for the color properties and $p_{k}$ are coefficients. The logistic function is often used in modeling, for example to describe the population growth and the failure rate of objects. This function could possibly be used to determine the mechanism of color change during heat treatment; in the present study, however, we just used it to describe and numerically analyze the color changes. Figure 2 shows the superposed data with the regression curve and $a_{T}$ at each treatment temperature. The regression curves fit well with the data set, as indicated by the coefficients of determination $\left(R^{2}\right)$ 0.996, 0.900, 0.861, and 0.995 for $L^{*}, a^{*}, b^{*}$, and $\Delta E^{*}$, , respectively. The regression for $b^{*}$ was slightly less accurate, as shown by the $R^{2}$ value and Fig. 2, because of variations in the data; it was, however, considered sufficient for analysis. The well-superposed curve implied that the color changes might be caused by the same process, irrespective of treatment temperatures. The apparent activation energy $\left(E_{a}\right)$ was calculated from the relationship in $\ln \left(a_{T}\right)$ and $1 / T$. The Arrhenius plots of $\Delta E^{*}{ }_{a b}$ and its linear regression line are shown in Fig. 3 as an example. Table 2 shows $E_{a}$ and $R^{2}$ for each parameter. The $E_{a}$ values calculated from the color properties $(95-117 \mathrm{~kJ} / \mathrm{mol})$ were similar to those obtained for other properties of wood such as weight reduction, MOE, and MOR (110-130 $\mathrm{kJ} / \mathrm{mol})[6,7]$. The regression lines showed a good linearity $\left(R^{2}>0.986\right)$, suggesting that the color change during heat treatment can be explained as thermal oxidation, irrespective of the treatment temperature. Kinetic analysis can therefore be applied to wood color properties and prediction of color change at temperatures at least up to $180^{\circ} \mathrm{C}$. 

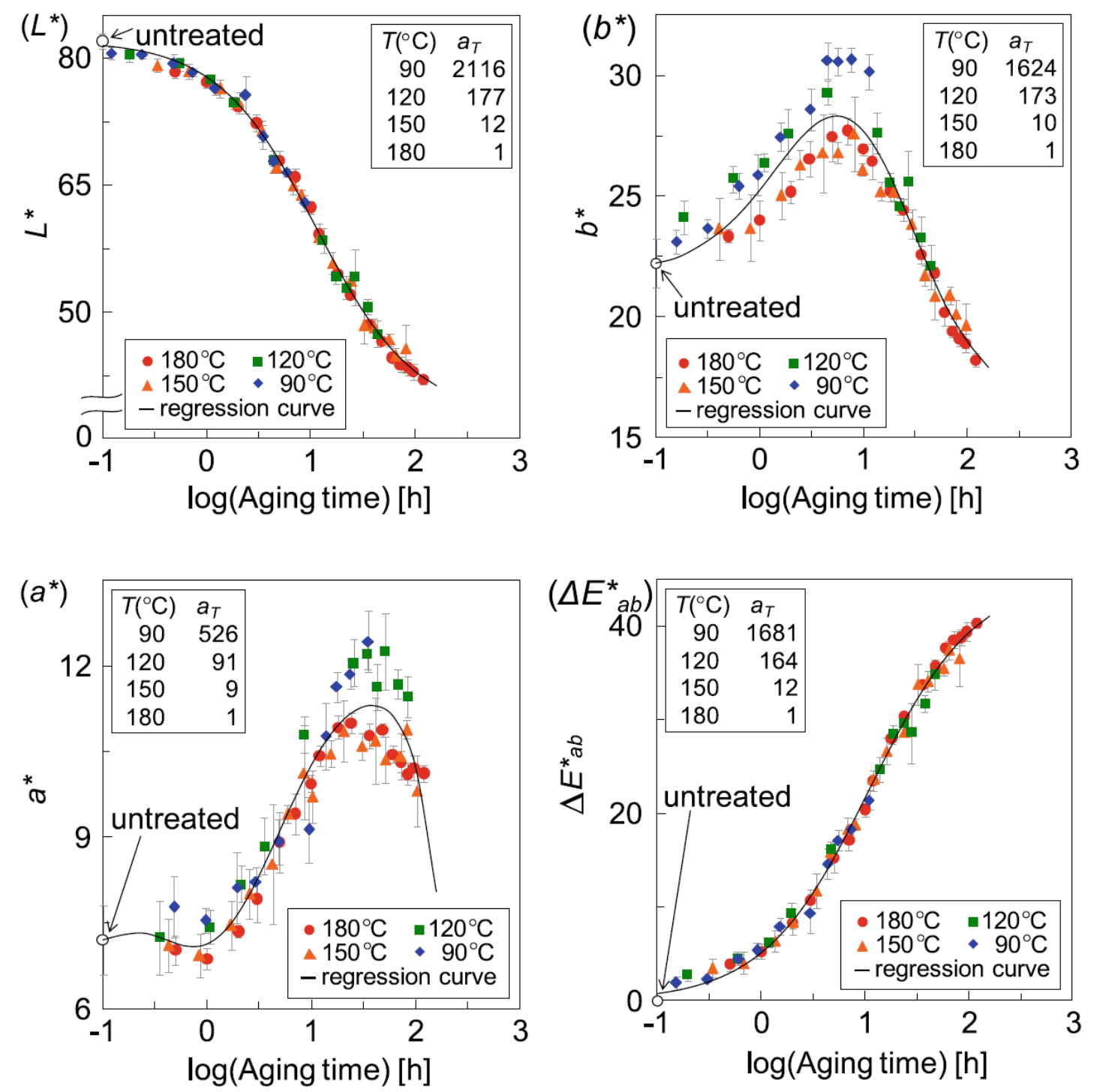

FIGURE 2 Superposed color properties with regression curves using $a_{T}$ at each temperature. $\left(\triangleleft: 90^{\circ} \mathrm{C}, \square: 120^{\circ} \mathrm{C}, \triangle: 150^{\circ} \mathrm{C}, \bullet: 180^{\circ} \mathrm{C}\right.$, error bar: standard deviation, $T$ : treatment time, $a_{T}:$ shift factor) 


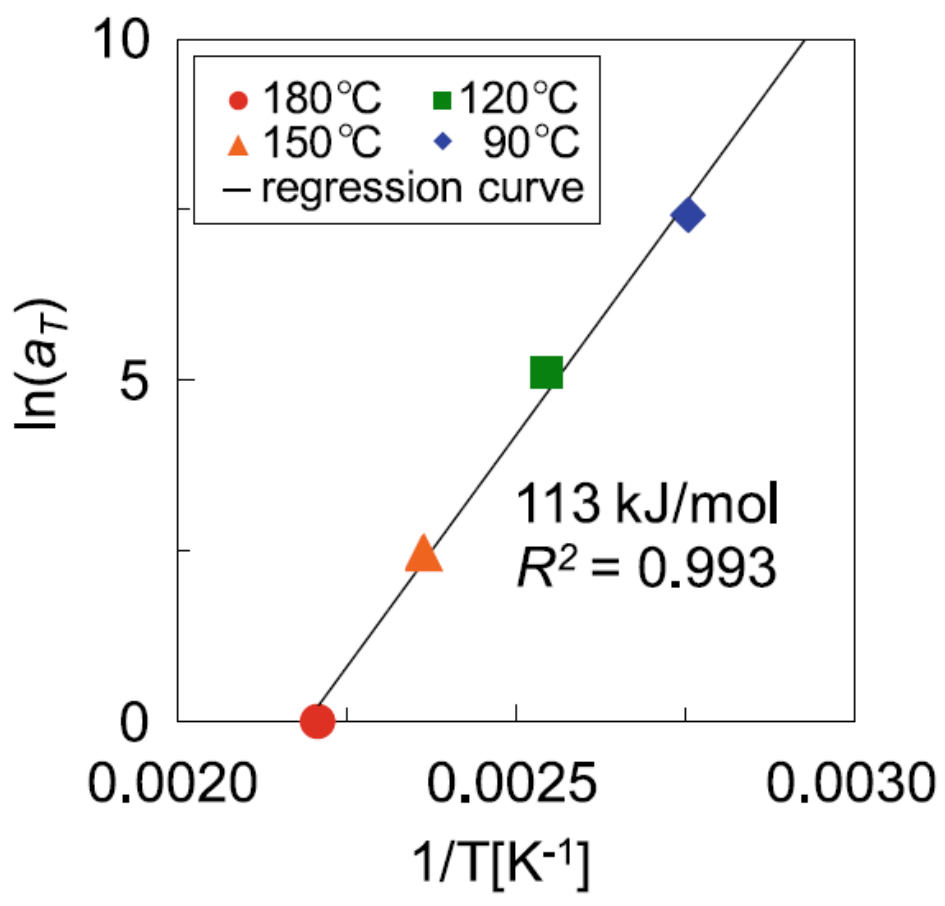

FIGURE 3 Arrhenius plots of shift factors from empirical superposition of the change of $\Delta E^{*}{ }_{a b}$. $\left(\diamond: 90^{\circ} \mathrm{C}, \square: 120^{\circ} \mathrm{C}, \triangle: 150^{\circ} \mathrm{C}, \bullet: 180^{\circ} \mathrm{C}\right.$, error bar: standard deviation)

TABLE 2 Apparent activation energies $\left(E_{a}\right)$ and coefficients of determination $\left(R^{2}\right)$ estimated from the Arrhenius plots of color properties.

\begin{tabular}{lcl}
\hline & $E_{a}[\mathrm{~kJ} / \mathrm{mol}]$ & $R^{2}$ \\
\hline$L^{*}$ & 117 & 0.995 \\
$a^{*}$ & 95 & 0.986 \\
$b^{*}$ & 114 & 0.993 \\
$\Delta E^{*}{ }_{a b}$ & 113 & 0.993 \\
\hline
\end{tabular}

\section{Conclusions}

Color changes of hinoki wood heated at $90-180^{\circ} \mathrm{C}$ were measured to evaluate color changes during accelerated aging. The values of $L^{*}$ decreased and $\Delta E^{*}{ }_{a b}$ increased and the values of $a^{*}$ and $b^{*}$ initially increased and then decreased over all the treatment temperatures. Kinetic analysis was applied successfully to describe the color changes. According to the kinetic analysis using the TTSP method, which allows for the use of whole data, the color change occurring during heat treatment could be explained as originating in thermal oxidation. The apparent activation energy calculated 
from the color properties was $95-117 \mathrm{~kJ} / \mathrm{mol}$. These values were close to the values calculated from other properties such as various mechanical properties and weight decrease. Our results indicate that the color changes occurring due to thermal oxidation during natural aging can be predicted by kinetic analysis.

\section{Acknowledgements}

This work was supported by Grant-in-Aid for Scientific Research (No. 20248020) from the Japan Society for the Promotion of Science.

\section{References}

1. J.J.M. Órfão, J.L. Figueiredo: Thermochimica Acta 380, 67 (2001)

2. M.J. Prins, K.J. Ptasinski, F.J.J.G. Janssen: J. Anal. Appl. Pyrolysis 77, 28 (2006)

3. M. Müller-Hagedorn, H. Bockhorn: J. Anal. Appl. Pyrolysis 79, 136 (2007)

4. C.D. Blasi: Progress in Energy and Combustion Sci. 34, 47 (2008)

5. T. Fisher, M. Hajaligol, B. Waymack, D. Kellogg: J. Anal. Appl. Pyrolysis 62, 331 (2002)

6. A.J. Stamm: Ind. and Eng. Chem. 48(3), 413 (1956)

7. M.A. Millett: Wood Sci. 4(4), 193 (1972)

8. X. Zou, T. Uesaka, N. Gurnagul: Cellulose 3, 243 (1996)

9. X. Zou, T. Uesaka, N. Gurnagul: Cellulose 3, 269 (1996)

10. J. Łojewska, A. Lubańska, P. Miśkowiec, T. Łojewski, L.M. Proniewicz: Appl. Phys. A 83, 597 (2006)

11. B.M. Esteves, H.M. Pereira: BioResources 4(1), 370 (2009)

12. J.A. Steet, C.H. Tong: J. Food Sci. 61(5), 924 (1996)

13. I.M.L.B. Ávila, C.L.M. Silva: J. Food Eng. 39, 161 (1999)

14. A. Ibarz, J. Pagán, s. Garza: J. Food Eng. 39, 415 (1999)

15. C.A. Weemaes, V. Ooms, A.M.V. Loey, M.E. Hendrickx: J. Agric. Food Chem. 47, 2404 (1999)

16. P. Burtin, C.J. Allemand, J.P. Charpentier, G. Janin: Holzforschung 54, 33 (2000)

17. P. Bekhta, P. Niemz: Holzforschung 57, 539 (2003)

18. D. Johansson, T. Moren: Holz als Roh- und Werkstoff 64, 104 (2006)

19. C. Brischke, C.R. Welzbacher, K. Brandt, A.O. Rapp: Holzforschung 61, 19 (2007)

20. B.M. Esteves, A.V. Marques, I. Domingos, H.M. Pereira: Wood Sci. and Tech. 42, 369 (2008)

21. J. Kohara: Ind. and Eng., Chiba University 9(16), 23 (1958) [in Japanese]

22. J. Kohara: Ind. and Eng., Chiba University 9(15), 1 (1958) [in Japanese] 
Matsuo et al Applied Physics A, vol. 99, 1, 47-52. (Author's copy)

23. JIS Z 8729, 8730 (2006)

24. H.Z. Ding, Z.D. Wang: Cellulose 14(3), 171 (2007)

25. K.T. Gillen, R.L. Clough: Polymer Degradation and Stability 24, 137 (1988)

26. J. Wise, K.T. Gillen, R.L. Clough RL: Polymer Degradation and Stability 49, 403 (1995)

27. K.T. Gillen, M. Celina: Polymer Degradation and Stability 71, 15 (2001) 